\title{
Outcome of very premature infants with necrotising enterocolitis cared for in centres with or without on site surgical facilities
}

\author{
M Loh, D A Osborn, K Lui on behalf of the NSW Neonatal Intensive Care Unit Study \\ (NICUS) group
}

Royal Hospital for Women, Randwick, NSW, Australia $M$ Loh

Royal Prince Alfred Hospital,

Camperdown, NSW, Australia

D A Osborn

School of Paediatrics, University of New South Wales, Sydney, NSW, Australia

K Lui

Correspondence to: Dr Lui, Department of Newborn Care, Royal Hospital for Women, Barker Street, Randwick, New South Wales, Australia 2031 k.lui@unsw.edu.au

Accepted 3 April 2001

\begin{abstract}
Objective-To determine if the presence of a neonatal surgical facility on site has any effect on mortality and morbidity of very premature infants with necrotising enterocolitis (NEC).

Design and Setting-Retrospective review of infants of less than 29 weeks gestation cared for in the seven perinatal centres in New South Wales.

Patients-Between 1992 and 1997, 605 infants were cared for in two centres with in house surgical facilities (group 1) and 1195 in five centres where transfers were required for surgical management (group 2). Results-Although use of antenatal steroids was significantly lower in group 1 centres than group 2 centres $(74 \% v 85 \%$ respectively), and the incidence of hyaline membrane disease, pneumothorax, and NEC was higher, mortality was identical $(27 \%)$. Fifty two $(9 \%)$ infants in group 1 and $72(6 \%)$ in group 2 of comparable perinatal characteristics and CRIB (Clinical Risk Index for Babies) scores developed radiologically or pathologically proven NEC. The overall mortality of infants with NEC in group 1 was lower but this was not statistically significant $(27 \% v$ $35 \%)$. Significantly more infants with NEC in group 1 had surgery $(69 \% v 39 \%)$. There were fewer postoperative deaths in group 1 and more deaths without surgery in group 2. The duration of respiratory and nutritional support and hospital stay for the survivors were similar in the two groups. In a multivariate analysis, shorter gestation was the only factor associated with mortality in infants with NEC; the presence of in house surgical facilities was not. Conclusions-There were no significant differences in outcome of premature infants with NEC managed in perinatal centres with or without on site surgical facilities. Early transfers should be encouraged. This finding may have implications for future planning of facilities for neonatal care.
\end{abstract}

(Arch Dis Child Fetal Neonatal Ed 2001;85:F114-F118)

Keywords: necrotising enterocolitis; premature infants; transfer; mortality

Necrotising enterocolitis (NEC) is the commonest surgical emergency encountered in the neonatal period..$^{12}$ Most cases are found in premature infants, with incidence and mortality rising with decreasing gestation and birth weight..$^{3-5}$ Although many cases of NEC can be successfully managed medically, 30$60 \%$ ultimately require surgery. ${ }^{1-36-8}$ The improved survival of surgically treated infants with NEC of all gestational ages, currently more than $70 \%,{ }^{278}$ has been attributed to improved surgical and nursing expertise and experience in centres equipped for neonatal surgery.

However, not all neonatal intensive care units (NICUs) have in house surgical facilities. Current opinion recommends a modest caseload to maintain expertise. ${ }^{9}$ Thus infants with NEC who need surgical care are transferred to a centralised neonatal surgical tertiary unit. Transport of any ill preterm infant can be problematic, with instability caused by handling having adverse effects on outcome. ${ }^{10}$ Bowman et $a l^{11}$ showed increased mortality in premature infants transferred between tertiary perinatal centres, but others did not agree. ${ }^{12}$ When, if at all, to transfer and operate on an ill infant with NEC is less clear. The potential risks of transfer have to be weighed against the surgical needs of the infant. ${ }^{13}$

The aim of this study was to compare the mortality and morbidity of very premature infants with NEC managed in centres with on site surgical facilities with similar infants in centres where transfer for surgery is necessary. The hypothesis is that management of such infants in surgical tertiary centres without the need of transport or a change in management team is associated with a better overall outcome.

\section{Patients and methods}

New South Wales (NSW) has seven tertiary perinatal centres providing neonatal intensive care. During most of the study period (19921997 inclusively), two centres had surgical facilities on site, one of which ceased to have in house surgical facilities from October 1995. Neonates needing surgical intervention were transported to either of the two above centres or to the neonatal facilities of the two children's hospitals for further management. All centres are university affiliated hospitals and have subspecialty trained neonatologists in attendance full time. All centres without surgical facilities, except one, have visiting paediatric surgeons from the two children's hospitals for consultation. Neonatal transport was conducted by either of the specialised teams from the two children's hospitals, and the services were 
Table 1 Comparison of all 1800 infants cared for in centres with on site surgical facilities (group 1) or in centres without (group 2)

\begin{tabular}{lllll}
\hline Variable & Group 1 & Group 2 & p Value & Odds ratio (95\% CI) \\
\hline No of infants & 605 & 1195 & & \\
Gestation (weeks) & $26.3(1.4)$ & $26.4(1.4)$ & 0.15 & \\
Birth weight (g) & $919(231)$ & $929(226)$ & 0.38 & \\
Male sex & $318(53 \%)$ & $613(51 \%)$ & 0.62 & $1.05(0.86$ to 1.28$)$ \\
Pre-eclampsia & $82(14 \%)$ & $175(15 \%)$ & 0.57 & $0.91(0.69$ to 1.21$)$ \\
Antepartum haemorrhage & $180(30 \%)$ & $357(30 \%)$ & 1.00 & $0.99(0.80$ to 1.23$)$ \\
Growth restriction & $34(6 \%)$ & $67(6 \%)$ & 1.00 & $1.00(0.66$ to 1.53$)$ \\
Antenatal corticosteroid & $449(74 \%)$ & $1016(85 \%)$ & $<0.0001^{\star}$ & $0.51(0.40$ to 0.65$)$ \\
Apgar at 5 min & $7.0(1.8)$ & $7.1(1.8)$ & 0.27 & \\
HMD & $483(80 \%)$ & $862(72 \%)$ & $0.0004^{\star}$ & $1.53(1.21$ to 1.94$)$ \\
Surfactant treatment & $362(60 \%)$ & $754(63 \%)$ & 0.18 & $0.87(0.71$ to 1.07$)$ \\
Pneumothorax & $85(14 \%)$ & $120(10 \%)$ & $0.02^{\star}$ & $1.46(1.09$ to 1.97$)$ \\
Proven NEC & $52(9 \%)$ & $72(6 \%)$ & $0.048^{\star}$ & $1.47(1.01$ to 2.13$)$ \\
IVH (grade 3-4) & $61(10 \%)$ & $128(11 \%)$ & 0.75 & $0.94(0.68$ to 1.29$)$ \\
Death & $162(27 \%)$ & $324(27 \%)$ & 0.91 & $0.98(0.79$ to 1.23$)$ \\
Steroid for CLD & $205(34 \%)$ & $434(36 \%)$ & 0.32 & $0.90(0.73$ to 1.10$)$ \\
ROP (grade 3-4) & $85(14 \%)$ & $19(9 \%)$ & $0.002^{\star}$ & $1.63(1.20$ to 2.20$)$ \\
Ventilation (days) & $15.0(17.5)$ & $17.7(20.0)$ & $0.003^{\star}$ & \\
Oxygen (days) & $47.1(49.0)$ & $40.4(45.6)$ & $0.005^{\star}$ & \\
TPN (days) & $14.1(16.9)$ & $13.9(14.0)$ & 0.80 & \\
Hospital stay (days) & $59.5(49.4)$ & $61.5(44.6)$ & 0.40 & \\
\hline
\end{tabular}

Values are mean (SD) or number (\%).

${ }^{\star} \mathrm{p}<0.05$.

HMD, Hyaline membrane disease; NEC, necrotising enterocolitis; IVH, intraventricular haemorrhage; CLD, chronic lung disease; ROP, retinopathy of prematurity; TPN, total parenteral nutrition.
Statistical Package for Social Science (SPSS) PC version 9.0 was used for statistical analysis.

\section{Results}

Of the 1800 preterm infants born at less than 29 weeks gestation during the study period, 605 were cared for in centres with surgical facilities on site and 1195 in centres without surgical facilities. The infant population and hospital outcome were similar in the two groups of centres. The two populations of infants were similar in terms of frequency of antenatal problems and they had similar mean gestational ages and birth weights (table 1). However, infants born at surgical perinatal centres received antenatal steroids less often than similar infants in non-surgical centres $(74.2 \%$ v $85 \% ; \mathrm{p}<0.0001)$. These infants had a higher incidence of hyaline membrane disease (HMD) $(79.8 \% v 72.1 \%$; $=0.0004)$ and pulmonary air leak $(14 \%$ v $10 \%$, $\mathrm{p}=0.015)$. The incidence of proven NEC was significantly higher among infants in group 1 $(9 \% v 6 \%, p=0.048)$. In spite of these differences, the overall hospital mortality was identical $(27 \%)$. On average, infants in group 1 spent less time receiving positive pressure ventilation $(\mathrm{p}=0.003)$, but more time in supplemental oxygen $(p=0.005)$. Severe grades of retinopathy of prematurity (ROP) were more common among infants in group $1(p=0.002)$, but not severe intraventricular haemorrhage (IVH grade 3 or 4 ) or chronic lung disease (CLD) requiring treatment with postnatal steroids.

A total of 128 premature infants with a discharge diagnosis of proven NEC were reviewed. Four infants (two from each group) were excluded from this study for the following reasons. One was diagnosed as having haematochezia, but had no radiological evidence of NEC. Three other infants, on close scrutiny of medical records, had insufficient clinical evidence of NEC and no evidence of pneumatosis intestinalis radiologically. All four infants survived. The remaining 124 infants were included in the study.

The incidence of NEC in this infant population of less than 29 weeks gestation was thus $6.9 \%$. The mean (SD) gestation and birth weight were 26.2 (1.4) weeks and 894 (231) g respectively. Fifty two infants with NEC were cared for in the two tertiary centres with surgical facilities (group 1), and the remaining 72 were cared for in centres where transfer for surgery was required (group 2). Table 2 summarises the perinatal characteristics. The CRIB scores were similar in the two groups of infants. The use of antenatal steroids was lower in infants in group 1, but this difference did not reach significance $(71 \% v 86 \%, \mathrm{p}=0.07)$. The incidence of air leaks was higher in infants in group $1(21.2 \% v 4.2 \%, \mathrm{p}=0.004)$, but the incidence of HMD, use of surfactant, and treatment of patent ductus arteriosus were similar for the two groups.

Table 3 summarises clinical variables relating to NEC and its outcome in the two groups of premature infants. Most of the infants in both groups were fed before the onset of NEC. 
Table 2 Comparison of early perinatal characteristics in infants with necrotising enterocolitis cared for in centres with on site surgical facilities (group 1) and in centres without (group 2)

\begin{tabular}{lllll}
\hline Variable & Group 1 & Group 2 & p Value & Odds ratio (95\% CI) \\
\hline No of infants & 52 & 72 & & \\
Gestation (weeks) & $26.2(1.3)$ & $26.2(1.4)$ & 1.00 & \\
Birth weight (g) & $887(241)$ & $889(225)$ & 0.96 & \\
Male sex & $26(50 \%)$ & $40(55 \%)$ & 0.80 & $0.59(0.39$ to 1.64$)$ \\
Pre-eclampsia & $6(12 \%)$ & $6(8 \%)$ & 0.77 & $1.21(0.38$ to 3.84$)$ \\
Antepartum haemorrhage & $19(37 \%)$ & $18(25 \%)$ & 0.23 & $1.73(0.79$ to 3.76$)$ \\
Growth restriction & $6(12 \%)$ & $6(8 \%)$ & 0.56 & $1.44(0.44$ to 4.73$)$ \\
Antenatal corticosteroids & $37(71 \%)$ & $62(86 \%)$ & 0.07 & $0.40(0.23$ to 1.08$)$ \\
Apgar at 5 min & $7.0(1.7)$ & $7.2(1.7)$ & 0.52 & \\
CRIB score & $5.9(3.7)$ & $5.8(4.0)$ & 0.89 & \\
HMD & $39(75 \%)$ & $59(82 \%)$ & 0.38 & $0.66(0.28$ to 1.58$)$ \\
Surfactant treatment & $31(60 \%)$ & $45(63 \%)$ & 0.85 & $0.89(0.43$ to 1.84$)$ \\
Pneumothorax & $11(21 \%)$ & $3(4 \%)$ & $0.004 \star$ & $6.17(1.63$ to 23.43$)$ \\
Indomethacin for PDA & $20(39 \%)$ & $19(26 \%)$ & 0.17 & $1.74(0.81$ to 3.75$)$ \\
PDA surgery & $7(14 \%)$ & $4(6 \%)$ & 0.20 & $2.64(0.73$ to 9.56$)$ \\
IVH (grade 3-4) & $3(6 \%)$ & $8(11 \%)$ & 0.36 & $0.49(0.12$ to 1.94$)$ \\
\hline
\end{tabular}

Values are mean (SD) or number (\%).

${ }^{\star} \mathrm{p}<0.05$.

HMD, hyaline membrane disease; PDA, patent ductus arteriosus; IVH, intraventricular haemorrhage.

The age at first feed and NEC onset were similar between the groups. The rates of blood culture positive septicaemia in association with NEC were similar. On comparing haematological indices for severity of NEC, the lowest platelet count was lower in group 1 than group 2 infants $\left(114 \times 10^{9} v 188 \times 10^{9} / 1\right.$ respectively, $\mathrm{p}=0.02)$. The lowest neutrophil count during NEC was, however, lower in group 2 infants $\left(8.0 \times 10^{9}\right.$ v $\left.4.4 \times 10^{9} / 1, \mathrm{p}=0.01\right)$. The two groups of infants had similar rates of mechanical ventilation and correction of acidosis with intravenous bicarbonate as a result of NEC, but the infants in group 1 received inotropic support more often $(59 \% v 32 \%, \mathrm{p}=0.01)$.

The overall mortality was not significantly different between the two groups $(27 \%$ in group 1 infants and $35 \%$ in group 2) and averaged $31.5 \%$ for the whole population. Thirty eight of the 52 infants in group 1 had surgery: $36(69 \%)$ during acute NEC, one of whom had

Table 3 Comparison of clinical course and outcome of infants with necrotising enterocolitis (NEC) cared for in centres with on site surgical facilities (group 1) and in centres without (group 2)

\begin{tabular}{|c|c|c|c|c|}
\hline Variables & $\begin{array}{l}\text { Group } 1 \\
(n=52)\end{array}$ & $\begin{array}{l}\text { Group } 2 \\
(n=72)\end{array}$ & $p$ Value & Odds ratio $(95 \% \mathrm{CI})$ \\
\hline \multicolumn{5}{|l|}{ NEC clinical course } \\
\hline Age at onset of NEC (days) & $23.3(13.6)$ & $25.7(17.0)$ & 0.39 & \\
\hline Fed before NEC & $47(90 \%)$ & $65(90 \%)$ & 1.00 & $1.01(0.30$ to 3.39$)$ \\
\hline Age at first feed (days) & $7.0(4.7)$ & $7.4(5.2)$ & 0.66 & \\
\hline Proven sepsis with NEC & $16(31 \%)$ & $25(35 \%)$ & 0.70 & $0.84(0.39$ to 1.79$)$ \\
\hline Lowest platelet $\left(\times 10^{9} / 1\right)$ & $114(98)$ & $176(188)$ & $0.02^{\star}$ & \\
\hline Lowest WCC $\left(\times 10^{9} / 1\right)$ & $8.0(9.1)$ & $4.4(4.9)$ & $0.01^{\star}$ & \\
\hline Ventilated for NEC & $49(94 \%)$ & $63(88 \%)$ & 0.36 & 2.33 (0.60 to 9.08$)$ \\
\hline Inotropes for NEC & $29(56 \%)$ & $23(32 \%)$ & $0.01^{\star}$ & $2.69(1.28$ to 5.62$)$ \\
\hline Bicarbonate for acidosis & $7(14 \%)$ & $13(18 \%)$ & 0.62 & $0.71(0.26$ to 1.91$)$ \\
\hline Acute NEC surgery & $36(69 \%)$ & $28(39 \%)$ & $0.001^{\star}$ & 3.53 (1.66 to 7.53$)$ \\
\hline Deaths & $14 / 52(27 \%)$ & $25 / 72(35 \%)$ & 0.43 & $0.69(0.32$ to 1.51$)$ \\
\hline Died after acute surgery & $11 / 36(31 \%)$ & $14 / 28(50 \%)$ & 0.13 & $0.44(0.16$ to 1.22$)$ \\
\hline Died w/o surgery & $3 / 52(6 \%)$ & $11 / 72(17 \%)$ & 0.15 & 0.34 (0.09 to 1.28$)$ \\
\hline Survivors & 38 & 47 & & \\
\hline Resection for stricture & $3(8 \%)$ & $1(2 \%)$ & 0.32 & $3.94(0.39$ to 39.5$)$ \\
\hline CLD at 36 weeks & $15 / 38(39 \%)$ & $21 / 47(45 \%)$ & 0.66 & $0.80(0.34$ to 1.92$)$ \\
\hline Steroid for CLD & $18 / 38(47 \%)$ & $30 / 47(63 \%)$ & 0.19 & $0.51(0.21$ to 1.22$)$ \\
\hline Ventilation (days) & $27.4(16.7)$ & $33.5(28.1)$ & 0.22 & \\
\hline Oxygen (days) & $58.8(32)$ & $61.3(58.4)$ & 0.80 & \\
\hline ROP (grade 3-4) & $9 / 38(24 \%)$ & $8 / 47(17 \%)$ & 0.58 & $1.51(0.52$ to 4.40$)$ \\
\hline TPN (days) & $37(21)$ & $32.6(20.3)$ & 0.33 & \\
\hline Hospital stay (days) & $93.9(30.2)$ & $97.6(47.7)$ & 0.67 & \\
\hline
\end{tabular}

Values are mean (SD) or number (\%).

${ }^{\star} \mathrm{p}<0.05$.

WCC, White blood cell count; CLD, chronic lung disease; ROP, retinopathy of prematurity; TPN, total parenteral nutrition. subsequent resection for intestinal stricture. A further two had stricture resection after initially successful medical management. Fourteen infants died, 11 after surgery during acute NEC and three without surgery. Thirty four $(47 \%)$ of the 72 infants in group 2 were transferred to surgical centres. Not all transferred infants had surgery: six did not and one of them died. Of the 28 infants who had surgery, 13 died after the operation. One surgically treated survivor needed further resection for intestinal stricture. Of the 38 infants in group 2 who were not transferred, 10 died. The overall rate of surgery for NEC was significantly higher among infants in group $1(73 \%$ v $39 \%$, $\mathrm{p}=0.0002)$. Group 1 infants showed a trend towards a lower mortality after surgery, and more group 2 infants died without surgery (table 3). The duration of supplemental oxygen, mechanical ventilation, total parenteral nutrition, and hospital stay were similar for the survivors in the two groups.

Multivariate analysis using backward stepwise logistic regression based on likelihood ratio was performed for the 124 infants with NEC. Variables entered were gestation, birth weight, CRIB score, antenatal steroids, HMD, and group designation, with death as the outcome variable. Gestation was the only significant variable after elimination steps. The final model was constructed with three variables: gestation with antenatal steroid and group designation as forced entries. Gestation appeared to be the only independent factor that was close to statistical significance (odds ratio (OR) 0.77 per week increment, 95\% confidence interval $(95 \% \mathrm{CI}) \quad 0.57$ to 1.04$)$. Neither antenatal steroids (OR $1.39,95 \%$ CI 0.50 to 3.84 ) nor the centre of care (group 2: OR $1.33,95 \%$ CI 0.51 to 3.10 ) affected mortality independently.

\section{Discussion}

This study is the first population study to compare outcomes in management of NEC in premature infants between tertiary perinatal centres with surgical facilities and those without. It also seeks to establish the possible effects of transport on outcomes in ill premature infants with NEC. The incidence of NEC at $6.9 \%$ in this premature population is comparable to that of other studies..$^{2-5}$ However, the average age of NEC onset of 25 days was higher than in previous series. ${ }^{25}$ This is probably related to the short gestation of our study population. ${ }^{34}$

We found that the overall infant population was similar in the two groups of perinatal centres, and the neonatal outcomes were comparable. Most of the important variables that affect mortality, such as gestational age, birth weight, and CRIB score, were well matched in the infants with NEC in the two groups of perinatal centres, but a few differences were present as a result of individual centre circumstances or practices. These differences were not confined to infants with NEC but were representative of the respective infant populations. The most notable difference was the lower use of antenatal steroids in infants in 
group 1. This could account for the larger number of infants with HMD and pneumothorax in this group. The use of antenatal steroids may decrease the chances of developing NEC in infants at risk, and our findings that infants in group 2 had a lower incidence of NEC are consistent with those of Halac and colleagues. ${ }^{17}$ Whether this has a bearing on the severity of NEC and its effect on mortality is not known. However, when use of antenatal steroids was added to the multivariate analysis, it was found not to affect mortality in this NEC cohort.

In general, the characteristics of NEC at onset were similar in the two groups of infants in terms of postnatal age, feeding before NEC, and the need for supportive medical treatment. Infants in group 1 had a higher rate of inotropic support and surgical intervention. This may reflect greater severity of NEC in these infants or individual practice variation. NEC surgery at a rate of $73 \%$ is high, but our population is more premature than in other studies. ${ }^{3478}$ Infants in group 1 had lower platelet counts. This may have been due to the higher incidence of surgery. Those in group 2 had lower leucocyte counts and more died without surgery. This may also reflect the fact that not having neonatal surgical facilities on hand may influence the decision for transfer of infants with critical NEC for surgery, aggressive medical management being used instead. When and how to operate on a neonate with NEC is still the subject of much discussion, ${ }^{16}$ even more so if there is a lack of surgical facilities on site. Primary peritoneal drainage is only an adjunct to resuscitation and usually requires the skills of a surgeon, because a lack of response within 24 hours must still lead to operative laparotomy. ${ }^{6}$ More infants with NEC in surgical centres had surgery during the acute phase, and this was not offset by subsequent surgery for strictures in the infants from non-surgical centres. This observation may be explained by the fact that surgeons have a lower threshold for surgery than referring neonatologists in non-surgical centres. Despite a trend towards lower mortality after surgery in surgically equipped perinatal centres, the overall mortality was not significantly different. For survivors of NEC, the duration of subsequent respiratory and parenteral nutritional support in intensive care was similar in the two groups.

The delivery of babies with antenatally diagnosed surgical problems should probably be arranged to be in surgical perinatal centres or colocated obstetric and children's hospitals. However, NEC is a common surgical problem that arises suddenly and quickly in premature infants in the neonatal period. Although the possible complications of interhospital transfer of ill children have been emphasised, ${ }^{9}$ the risk of transferring such infants between level III centres is not clear. ${ }^{11} 1218$ Our findings contrast with those of Bowman and colleagues. ${ }^{11}$ The transfer of premature infants with NEC, whether they subsequently had surgery or not, did not increase the overall mortality in group 2 infants. Harding and Morton ${ }^{12}$ felt that perinatal outcomes depend more on the centre of care than the possible adverse effects of transfer. Our study showed that the group of centres, whether or not they needed to transfer to surgical facilities, did not independently influence mortality. In fact, decreasing gestational age was the only factor seen to affect mortality. This is consistent with the findings of other studies. ${ }^{35}$

The NICUs in our population study are all tertiary centres. It was recently shown that mortality in Australian NICUs was lower than in Scottish units. This was attributed to better medical and nursing staffing. ${ }^{19}$ In NSW, this is further supplemented with a specialised retrieval service. ${ }^{14}$ Our efficient perinatal services may account for the lack of differences in the mortality and morbidity examined. Planners of perinatal facilities have to ask whether it is safer and more cost effective to transfer infants with NEC to surgical centres or plan to have surgical facilities brought to such infants at risk on site. Our results show that there is no significant difference in NEC outcomes between the two models of care in NSW.

On the other hand, infants cared for in centres with surgical facilities had a non-significant trend towards lower overall mortality $(27 \% v$ $35 \%$ ) despite receiving less antenatal steroids and having a higher incidence of NEC. It is possible that statistical significance would be found with a larger sample size. Ten infants who were not transferred and one who was transferred died without surgery, in contrast with only three infants in surgical centres who died without surgery. The infants who were not transferred may have been judged to be too ill for transfer and may have survived if surgical facilities were on site. Half of the transferred infants who had surgery died. Therefore, it is also possible that infants became more unstable and ill after the transfer leading to a trend to higher postoperative mortality.

However, the trend toward lower mortality may not be practically manifest if future planning were to include surgical facilities for all tertiary perinatal centres or even to have a "mobile" surgical team to perform the operation. The concern in balancing harm and benefit is that such decentralisation of specialist surgical services would dilute clinical experience to the point where any benefits would be offset. Specialist paediatric services also depend on other specialties for support such as paediatric radiology, paediatric anaesthesia, pathology, and specialist nursing. All these require the concentration of expertise and facilities for optimal training of the duty medical and nursing staff, development of support services, and ultimately better results. ${ }^{9}$ The availability of a specialised paediatric emergency transport service would also support the benefits of centralisation. One could recommend that these very premature infants with NEC be transferred as early as practical. This approach is justified because of the high mortality and need for surgical intervention.

Important questions remain about the long term growth and developmental outcome of the survivors, which were not examined in this comparative study. Our previous study ${ }^{20}$ from a 
centre with on site surgical facilities has emphasised the need for long term follow up. Increased neurodevelopment morbidity was shown in infants with NEC who required surgery compared with gestation matched controls without NEC and medically treated infants with NEC. Therefore, a further study to compare long term outcome in these two models of care is required.

In conclusion, we find that management of premature infants with NEC in perinatal centres where transfer is required for surgery is not significantly associated with increased mortality. We also find that management of premature infants with NEC in perinatal centres with surgical facilities on site is not necessarily associated with a better outcome. This is reassuring for the current model of neonatal care and transfer in NSW, and may help influence planning of perinatal facilities in the future.

We thank the directors, the NICUS members, and audit officers We thank the directors, the NICUS members, and audit officers of all tertiary units in supporting this collaborative study and
assistance in retrieval of medical records for review: NICUS, Dr assistance in retrieval of medical records for review: NICUS, Dr
Lee Sutton (Director), Barabara Bajuk (Coordinator); John Lee Sutton (Director), Barabara Bajuk (Coordinator); John Hunter Hospital, Dr Andrew Gill (Director), Michelle Giles; King George V Hospital, Dr Nick Evans (Director), Dr Phil Beeby, Shelley Reid; Liverpool Hospital, Dr Robert Guaran
(Director), Dr Ian Callendar, Dr Jacqueline Stack, Catherine (Director), Dr Ian Callendar, Dr Jacqueline Stack, Catherine Medlin, Sara Wilson; Nepean Hospital, Dr Lynnette Downs (Director), Mee Fong Chin; New Children's Hospital, Dr Robert Halliday (Director), Dr Nadia Badawi, Sharon Laing; Royal North Shore Hospital, Dr Jenny Bowen (Director), Dr Martin Kluckow, Pamela Hand; Sydney Children's Hospital, Dr Barry Duffy (Director), Janelle Young; Westmead Hospital, Dr Marilyn Rochefort (Director), Jane Baird; and Royal Hospital for Women, Dr Kei Lui (Director), Dr Paul Garvey, Dianne Cameron. We also thank Professor David Henderson-Smart, Director, NSW Pregnancy and Newborn Services Network for his advice.

1 Cikrit D, Mastandrea T, West KW, et al. Necrotising enterocolitis: factors affecting mortality in 101 cases. Surgery 1984;96:648-55

2 Grosfeld JL, Cheu H, Schlatter M, et al. Changing trends in necrotising enterocolitis: experience with 302 cases in two decades. Ann Surg 1991;214:300-6.
3 Beeby PJ, Jeffery H. Risk factors for necrotising enterocolitis: the influence of gestational age. Arch Dis Child 1992;67:432-5.

4 Lui K, Nair A, Giles W, Morris J, et al. Necrotising enterocolitis in a perinatal centre. 7 Paediatr Child Health 1992;28:47-9.

5 Palmer SR, Biffin A, Gamsu HR. Outcome of neonatal necrotising enterocolitis. Results of the BAPM/CDSC surveillance study 1981-84. Arch Dis Child 1989;4:388-94.

6 Cheu HW, Sukarochana K, Lloyd DA. Peritoneal drainage for necrotising enterocolitis. F Paediatr Surg 1988;23:55761 .

7 Jackman S, Brereton RJ, Wright VM. Results of surgical treatment of neonatal necrotising enterocolitis. Br f Surg 1990;77:146-8.

8 Ross MN, Wayne ER, Janik JS, et al. A standard of comparison for acute surgical necrotising enterocolitis. $\mathcal{F}$ Paediatr Surg 1989;24:998-1002.

9 Arul GS, Spicer RD. Where should paediatric surgery be performed? Arch Dis Child 1998;79:65-72.

10 Barry PW, Ralston C. Adverse events occurring during inter-hospital transfer of the critically ill. Arch Dis Child 1994;74:8-11.

11 Bowman E, Doyle LW, Murton LJ, et al. Increased mortality of preterm infants transferred between tertiary perinatal centres. BMF 1988;297:1098-100

12 Harding JE, Morton SM. Outcome of neonates transported between Level III centres. F Paediatr Child Health 1994;30:389-392.

13 Parigi GB, Bragheri R, Minniti S, et al. Surgical treatment of necrotising enterocolitis: when? How? Acta Paediatr 1994;396(suppl):58-61.

14 Rashid A, Bhuta T, Berry A. A regionalised transport service, the way ahead? Arch Dis Child 1999;80:488-92.

15 Tarnow-Mordi WO, Parry G, Moore J. The CRIB score: a tool for assessing initial neonatal risk and comparing performance in neonatal intensive care unit. Lancet 1993;342:193-8.

16 Schober PH, Nasseri J. Risk factors and severity indices in necrotising enterocolitis. Acta Paediatr 1994;396(suppl):49-52.

17 Halac E, Halac J, Begue EF, et al. Prenatal and postnatal corticosteroid therapy to prevent necrotising enterocolitis: a controlled trial. F Pediatr 1990;117:132-8.

18 Harding JE, Morton SM. Adverse effects of neonatal transport between Level III centres. F Paediatr Child Health port between L

19 International Neonatal Network, Scottish Neonatal Consultants, Nurses Collaborative Study Group. Risk adjusted and population based studies of the outcome for high risk infants in Scotland and Australia. Arch Dis Child Fetal Neonatal Ed 2000;82:F118-23.

20 Tobiansky R, Lui K, Roberts S, et al. Neurodevelopmental outcome in very low birthweight infants with necrotizing enterocolitis requiring surgery. 7 Paediatr Child Health 1995;31:233-6. 